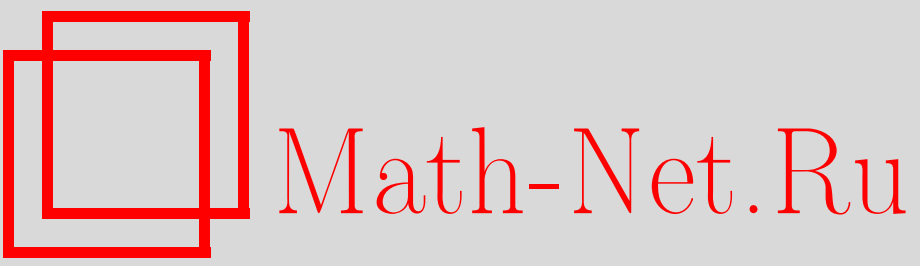

А. В. Астрелин, И. Х. Сабитов, Канонический многочлен для объема многогранника, УМН, 1999, том 54, выпуск 2, 165-166

DOI: https://doi.org/10.4213/rm136

Использование Общероссийского математического портала Math-Net.Ru подразумевает, что вы прочитали и согласны с пользовательским соглашением

http://www.mathnet.ru/rus/agreement

Параметры загрузки:

IP: 52.205 .19 .152

26 апреля 2023 г., 09:57:47 


\title{
КАНОНИЧЕСКИЙ МНОГОЧЛЕН ДЛЯ ОБЪЕМА МНОГОГРАННИКА
}

\author{
А. В. АСТРЕЛИН, И. Х. САБИТОВ
}

Пусть в $\mathbb{R}^{3}$ дан гомеоморфный сфере многогранник с треугольными гранями. Пусть $n$ - число его вершин, и пусть вершины пронумерованы в некотором порядке от 1 до $n$. Пусть $x=\left\{x_{1}, y_{1}, z_{1}\right.$, $\left.x_{2}, y_{2}, z_{2}, \ldots, x_{n}, y_{n}, z_{n}\right\}$ - координаты его вершин, выписанные в порядке нумерации вершин, $l=\left\{l_{1}, \ldots, l_{e}\right\}$-квадраты длин ребер, пронумерованных в некотором порядке от 1 до $e=3 n-6$; $V$ - объем, $l(x)$ и $V(x)$ - выражения $l$ и $V$ через координаты. Так как

$$
l_{k}=\left(x_{i}-x_{j}\right)^{2}+\left(y_{i}-y_{j}\right)^{2}+\left(z_{i}-z_{j}\right)^{2}, \quad 1 \leqslant k \leqslant e,
$$

где $(i, j)$ - номера вершин, соединенных ребром с номером $k$, и $V=\sum_{i} V_{i}$, где $V_{i}$ - ориентированный объем тетраэдра с вершиной в $(0,0,0)$ и с основанием на $i$-й грани многогранника, $1 \leqslant i \leqslant 2 n-4$, вычисляемый как смешанное произведение соответствующих векторов, то $l(x) \in \mathbb{Q}[x], V(x) \in \mathbb{Q}[x]$ (напомним, что запись вида $q \in \mathbb{Q}[x][y]$ означает, что $q$ является многочленом от $y$ с коэффициентами, полиномиально зависящими от $x$, в то время как запись $q \in \mathbb{Q}(x)[y]$ означает, что коэффициенты многочлена относительно $y$ зависят от $x$ как рациональные функции).

Ниже мы будем рассматривать многочлены относительно $V$; под степенью многочлена мы будем понимать его степень относительно $V$, под коэффициентом - коэффициент при определенной степени $V$ (как правило, он будет многочленом или рациональной функцией от $l$ или $x$ над полем рациональных чисел). Обозначим старший коэффишиент многочлена $Q \in \mathbb{Q}[l][V]$ или $Q \in \mathbb{Q}(l)[V]$ как $l c(Q)$.

Известно [1], что для любого многогранника существует многочлен $P_{0} \in \mathbb{Q}[l][V]$ такой, что $l c\left(P_{0}\right)=1$ (т.е. $P_{0}$ имеет вид $P_{0}=V^{N}+\sum_{n<N} p_{n}(l) V^{n}$, где $p_{n}(l)$ - многочлены от $l$ ) и $P_{0}(l(x), V(x)) \equiv 0$. Этот многочлен позволяет, в частности, по известной метрике многогранника получить конечное множество чисел - корней многочлена, содержащее значения объемов всех реализаций (в том числе, комплексных) метрики этого многогранника. Но алгоритм построения этого многочлена таков, что он, во-первых, не однозначен и по нему можно построить много таких многочленов, во-вторых, он дает заведомо более высокую степень, чем должно быть в действительности (например, для октаэдра получается степень 1024, а в реальности минимальная степень равна 16). В этой работе мы даем однозначный способ построения требуемого многочлена минимальной степени.

Пусть $S$ - множество многочленов $Q \in \mathbb{Q}[l][V]$ таких, что $Q(l(x), V(x)) \equiv 0$, не обязательно со старшим коэффициентом 1 . Нижеследующая теорема описывает некоторые свойства этого множества.

Теорема. 1. Пусть $d$-наименьшая степень (ненулевых) многочленов из $S$. Тогда среди многочленов из $S$ степени $d$ найдется многочлен $Q_{0}$ такой, что $\operatorname{lc}\left(Q_{0}\right)=1$.

2. Многочлен $Q_{0}$ делит все многочлены из $S$.

3. Такой многочлен $Q_{0}$ единственный.

Сначала сделаем следующее замечание. В уравнениях (1), связывающих неизвестные координаты вершин многогранника топологического типа сферы с длинами его ребер, число неизвестных равно $3 n-6$, т.е. числу ребер (считаем, что многогранник закреплен как твердое тело), поэтому число ребер многогранника в точности равно числу степеней свободы вершин этого многогранника, не считая перемещений его как твердого тела. Следовательно, на длины ребер многогранника не накладьвается дополнительных ограничений типа равенства. Это значит, что если в любой ненулевой элемент $w(l) \in \mathbb{Q}(l)$ вместо переменных $l_{k}$ подставить их значения через $x$ по формуле (1), то получается многочлен $w(l(x)) \not \equiv 0$, или

$$
0 \not \equiv w(l) \in \mathbb{Q}[l] \Rightarrow w(l(x)) \not \equiv 0 .
$$

Работа выполнена при финансовой поддержке Российского фонда фундаменталњных исследований (гранты № № 96-01-00836, 99-01-00867). 
Действительно, по теореме Штейница, любой триангуляции сферы можно сопоставить выпуклый многогранник, комбинаторно изоморфный этой триангуляции, поэтому для любого гомеоморфного сфере многогранника с треугольными гранями существует изоморфный ему строго выпуклый многогранник. Пусть $x^{0}$ - точка, соответствующая вершинам этого строго выпуклого многогранника, а $l^{0}$ - набор квадратов длин ребер этого многогранника. В силу жесткости строго выпуклого многогранника отображение $x \rightarrow l, l=l(x)$, в окрестности точек $x^{0}$ и $l^{0}$ является диффеоморфизмом, поэтому существует обратное отображение $x=x(l)$. Значит, если $w(x)=w(l(x)) \equiv 0$, то $w(l)=w(x(l)) \equiv 0$, что противоречит условию $w(l) \not \equiv 0$.

Лемма. Пусть $P, Q \in S$ u $l c(P)=1$. Возьмем $R=$ НОД $(P, Q) \in \mathbb{Q}[V]$, для которого $l c(R)=1$ (такой многочлен существует, поскольку его коэффичиенты лежат в поле $\mathbb{Q}(l))$. Тогда:

a) $\operatorname{deg} R>0$; б) $R \in \mathbb{Q}[l][V]$.

ДокАЗАтЕльство леммы. а) Будем искать $R$ с помощью алгоритма Евклида, т.е. рассмотрим последовательность многочленов $T_{1}=P, T_{2}=Q, T_{n+1}=T_{n-1}-T_{n} U_{n}$, где $\operatorname{deg} T_{n+1}<\operatorname{deg} T_{n}, T_{n}, U_{n} \in \mathbb{Q}(l)[V], n>1$.

Параллельно рассмотрим последовательность $\widetilde{P}=\widetilde{T}_{1}=P(l(x), V), \widetilde{Q}=\widetilde{T}_{2}=Q(l(x), V)$, $\widetilde{T}_{n+1}=\widetilde{T}_{n-1}-\widetilde{T}_{n} \widetilde{U}_{n}$. Так как, согласно (2), неравные нулю старшие коэффициенты многочленов $T_{n}(l)$ превращаются в ненулевые коэффициенты и для $\widetilde{T}_{n}$ при тех же степенях $V$, то (по индукции) $\widetilde{T}_{k}=T_{k}(l(x), V)$, следовательно, $\widetilde{R}=\operatorname{HОД}(\widetilde{P}, \widetilde{Q})=R(l(x), V)$. Но $(V-V(x)) \mid \widetilde{P}$ (т.е. многочлен $V-V(x)$ делит многочлен $\widetilde{P}(x)[V]),(V-V(x)) \mid \widetilde{Q}$ в кольце $\mathbb{Q}(x)[V]$, значит, $(V-V(x)) \mid \widetilde{R}_{\text {и }} \operatorname{deg} R=\operatorname{deg} \widetilde{R}>0$.

б) Поскольку $R \mid P$, то $P=R W$, где $P \in \mathbb{Q}[l][V], R, W \in \mathbb{Q}(l)[V], l c(R)=l c(W)=1$. В этом случае $R, W \in \mathbb{Q}[l][V]$. Доказательство от противного: пусть какой-нибудь коэффициент $R$ или $W$ не принадлежит $\mathbb{Q}[l]$ и $s$ - какой-нибудь неприводимый делитель его знаменателя. Рассмотрим коэффициенты $r_{a}$ и $w_{b}$ многочленов $R$ и $W$, в которые $s$ входит в минимальной степени $t_{a}$ и $u_{b}$ соответственно (одно из этих чисел отрицательно, а другое неположительно); если таких коэффициентов несколько, выберем тот, что при минимальной степени $V$. Тогда многочлены $R$ и $W$ имеют представления вида

$$
\begin{aligned}
R & =V^{M}+s^{t_{1}} A_{1}(l) V^{M-1}+\cdots+s^{t_{a}} A_{a}(l) V^{M-a}+\cdots+s^{t_{M}} A_{M}(l), \\
W & =V^{N-M}+s^{u_{1}} B_{1}(l) V^{N-M-1}+\cdots+s^{u_{b}} B_{b}(l) V^{N-M-b}+\cdots+s^{u_{N-M}} B_{N-M}(l),
\end{aligned}
$$

где $t_{i} \geqslant t_{a}$ при $i<a$ и $t_{i}>t_{a}$ при $i>a ; u_{i} \geqslant u_{b}$ при $i<b$ и $u_{i}>u_{b}$ при $i>b$. Получаем, что в коэффициент многочлена $P$ при $V^{N-a-b}$ множитель $s$ входит в степени $t_{a}+u_{b}<0$, что противоречит условию $P \in \mathbb{Q}[l][V]$. Лемма доказана.

ДОКАЗАТЕЛЬСтво теОРемЫ. 1. Возьмем любой многочлен $Q \in S: \operatorname{deg} Q=d$. Рассмотрим $R=$ НОД $\left(P_{0}, Q\right) \in \mathbb{Q}(l)[V]$, для которого $l c(R)=1$. По лемме, $R \in \mathbb{Q}[l][V]$, а из условия $(V-V(x)) \mid R(l(x), V)$ (в кольце $\mathbb{Q}(x)[V])$ следует, что $R(l(x), V(x))=0$, т.е. $R \in S$. Кроме того, $\operatorname{deg} R \leqslant \operatorname{deg} Q$, следовательно, $\operatorname{deg} R=d$. Значит, $R$ - искомый многочлен $Q_{0}$.

2 . Возьмем любой многочлен $Q \in S$. Рассмотрим $R=\operatorname{HОД}\left(Q_{0}, Q\right) \in \mathbb{Q}(l)[V]$. Уже доказано, что $l c\left(Q_{0}\right)=1$. Аналогично п. 1 , имеем $R \in S, l c(R)=1$ и $\operatorname{deg} R \leqslant d$. Следовательно, $R=Q_{0}$, т.е. $Q_{0} \mid Q$.

3. Пусть в $S$ есть два многочлена $R_{1}, R_{2} \in \mathbb{Q}[l][V] \mathrm{c} \operatorname{deg} R_{1}=\operatorname{deg} R_{2}=d, l c\left(R_{1}\right)=$ $l c\left(R_{2}\right)=1$, которые делят все многочлены из $S$. Так как они делят также и друг друга, то это, очевидно, возможно только при их равенстве. Теорема доказана.

\section{СПИСОК ЛИТЕРАТУРЫ}

[1] Сабитов И. Х. // Фунд. прикл. матем. 1996. Т. 2. № 4. С. 1235-1246. 